\title{
Pyoderma gangrenosum with its subtype affecting oral mucosa pyostomatitis vegetans following skin melanoma surgical excision in a patient with ulcerative colitis: a case report
}

\author{
Anna Niezgoda, Agnieszka Białecka, Luiza Marek-Józefowicz, Ewa Skrzeczko-Kwela, Rafał Czajkowski \\ Chair of Dermatology, Sexually Transmitted Diseases and Immunodermatology, Nicolaus Copernicus University in Torun, \\ Faculty of Medicine in Bydgoszcz, Poland \\ Adv Dermatol Allergol 2018; XXXV (2): 212-216 \\ DOI: https://doi.org/10.5114/ada.2018.75246
}

Pyoderma gangrenosum (PG) is a disease which aetiology has been described only partially. In the course of the disease damage to blood vessels occurs secondarily to neutrophils infiltration into the affected site. Skin lesions may be located throughout the body, but lower limbs are predominantly affected. The primary lesions include papule, nodule, pustule, vesicle which transform into peripherally growing skin ulcerations. Pyoderma gangrenosum may coexist with multiple conditions including haematologic disorders, inflammatory bowel disease, rheumatoid arthritis and other autoimmune diseases and malignancies [1-3].

Pyostomatitis vegetans (PSV) is classified by many authors as a subtype of PG, which affects oral mucosa. The majority of PSV cases coincide with inflammatory bowel disease [4].

This paper describes a case of a 69-year-old patient, with a history of ulcerative colitis (UC). Due to UC, the patient participated in a clinical trial of a biologic drug in 2009-2010, but no detailed data on the treatment were available. The disease was complicated with several episodes of gastrointestinal bleeding. The last reported bleeding occurred 3 months before the admission to the Clinic of Dermatology. It resulted in hypovolemic shock and it was associated with the occurrence of mucosal lesions. The patient was admitted to the Clinic of Dermatology in Bydgoszcz because of minor oral mucosa erosions observed for 3 months preceding hospitalization. During physical examination a pigmentary lesion sized $40 \times 30 \mathrm{~mm}$ on the patient's back was observed. Clinical and dermoscopic characteristics of the lesion were typical of melanoma. Histopathological examination of the excised lesion confirmed melanoma pT1a (0.5 mm in Breslow scale) and complete ex- cision. Clinical and dermoscopic presentation of melanoma diagnosed in our patient is presented in Figure 1.

Shortly after the skin lesion had been excised, a rapidly spreading ulceration on the patient's right lower limb appeared. The ulceration was $8 \mathrm{~cm}$ in diameter, with a necrotic crust in the middle of the lesion and an elevated dark red edge. Moreover, single pustules, which initially appeared on the patient's face, trunk and limbs began to transform into minor ulcerations. Erosions of oral mucosa progressed to deep ulcerations with inflammation in adjacent areas. Additionally, mucosal pustules within the oral cavity could be observed. Pathergy reaction within the post-surgical wound was present. Skin lesions were accompanied by systemic symptoms such as fever, malaise and pain. Skin and mucosal lesions observed in the course of the disease are presented in Figure 2.

Laboratory tests performed at admission revealed an increased C-reactive protein (CRP) plasma concentration $(132 \mathrm{mg} / \mathrm{l})$, increased erythrocyte sedimentation rate $(85 \mathrm{~mm} / \mathrm{h})$, mild normocytic anaemia with haemoglobin concentration of $11.9 \mathrm{~g} / \mathrm{dl}$, and hypoalbuminaemia $3.28 \mathrm{~g} / \mathrm{dl}$. Microbiological examination of samples obtained from the surgical wound and thigh ulceration, as well as blood culture were negative. Additionally, only physiologic flora of the oral cavity was found in samples taken from oral mucosal ulcerations. Imaging examinations, including chest X-ray, abdominal ultrasound, peripheral lymph nodes ultrasound, paranasal sinuses computed tomography done during the hospitalization, revealed no significant abnormalities. A series of immunologic tests were performed, cytoplasmic anti-neutrophil cytoplasmic antibodies (c-ANCA) and perinuclear

Address for correspondence: Anna Niezgoda MD, Chair of Dermatology, Sexually Transmitted Diseases and Immunodermatology, Nicolaus Copernicus University, Faculty of Medicine, 9 Sklodowskiej-Curie St, 85-094 Bydgoszcz, Poland, phone: +48 606889 288, e-mail: anna.niezg@gmail.com Received: 19.04.2017, accepted: 31.07.2017. 

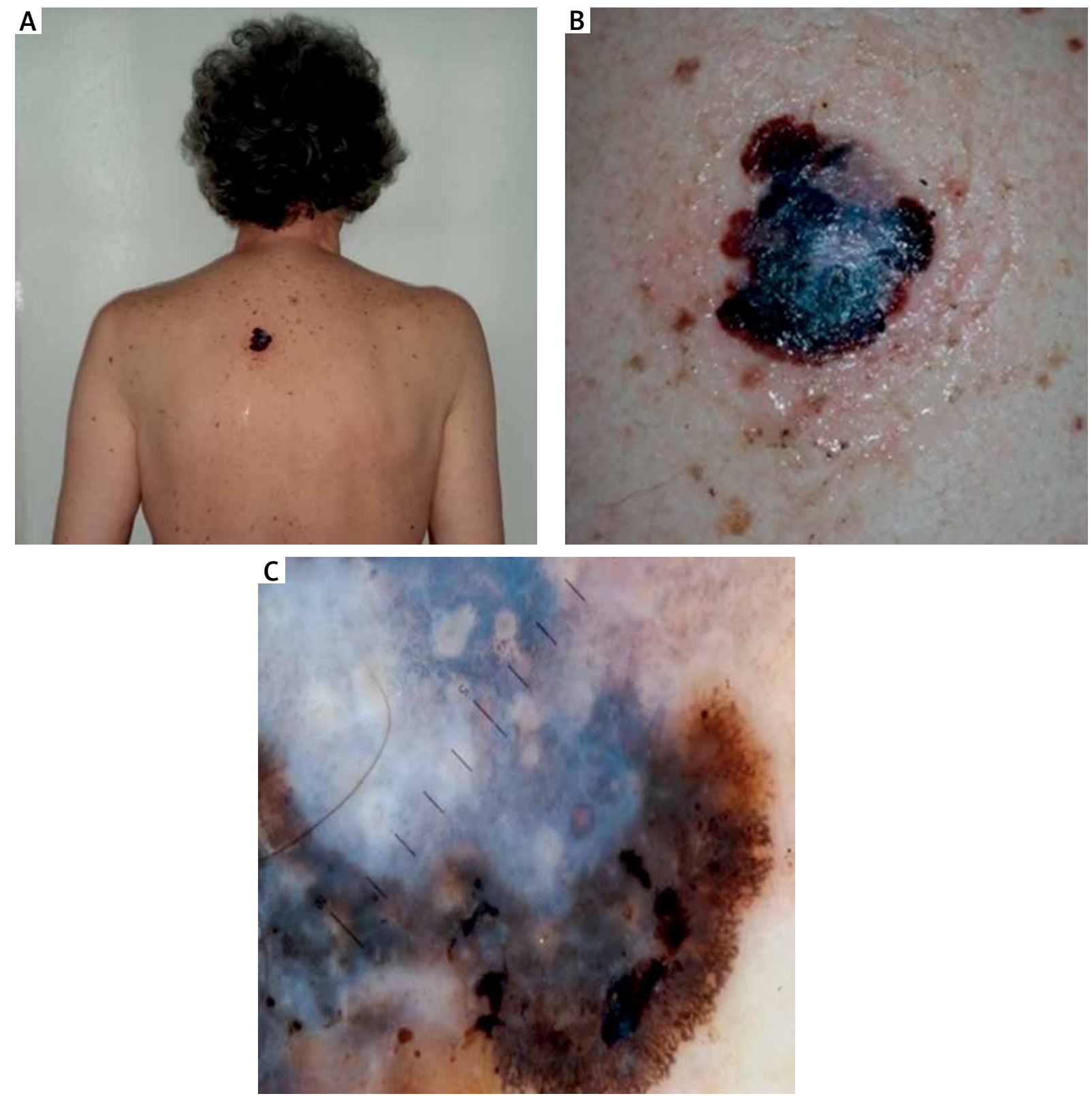

Figure 1. Clinical and dermoscopic presentation of melanoma

anti-neutrophil cytoplasmic antibodies ( $p$-ANCA) were negative, squamous epithelium-specific antibodies (SESANA), pemphigus and pemphigoid associated antibodies were negative. Oral mucosa direct immunofluorescence assay was negative. Anti-nuclear antibodies (ANA) of a homogenous and speckled pattern in titre $1: 640$ were found, whereas ANA EUROLINE profile 3 was negative. Ophthalmologic examination did not reveal traits of choroiditis or retinitis.

Initially, histopathological examination of oral mucosa revealed features of discreet keratosis, intraepithelial abscesses, reactive vascular proliferation within sub- epithelial connective tissue, erythrocyte extravasation with diffuse mononuclear cells influx (Figure $3 \mathrm{~A}$ ). When oral mucosal ulcerations appeared, the histopathological evaluation revealed massive eosinophilic, neutrophilic and lymphocytic infiltrate, acanthosis and eosinophil intravascular mobilization (Figure 3 B). Histopathological findings of the biopsy obtained from the edge of skin ulcerations included abundant suppurative infiltrate, necrosis with abscess formation and features of leukocytoclastic vasculitis (Figure $3 \mathrm{C}$ ).

Taking into consideration the overall clinical presentation as well as the results of laboratory tests and histo- 

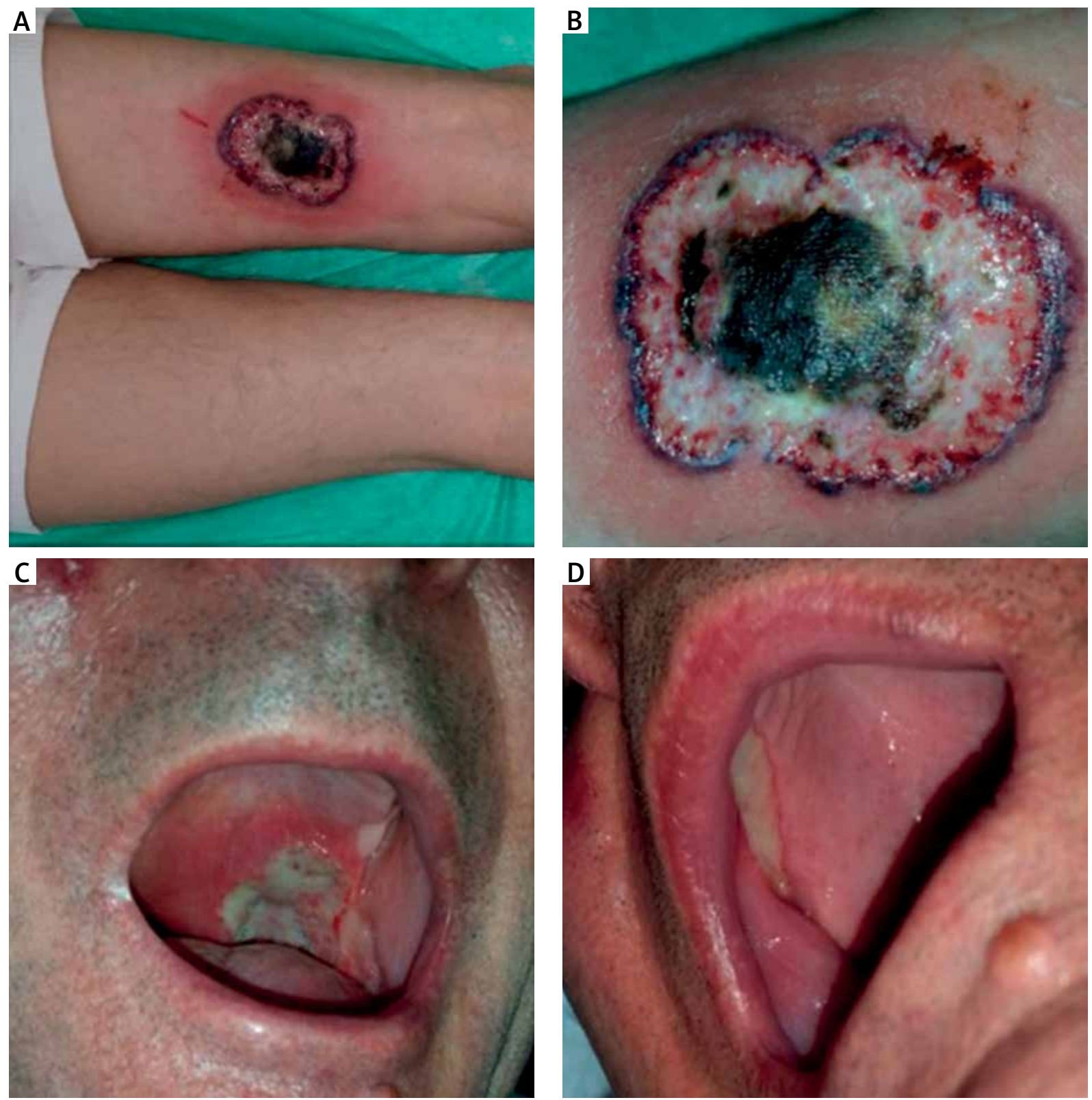

Figure 2. Skin and mucosal lesions observed in advanced phase of the disease

pathological examinations, the most probable diagnosis of PG and its subtype PSV was made.

The patient was initially treated with a combination of prednisone $40 \mathrm{mg}$ daily (0.6 $\mathrm{mg} / \mathrm{kg}$ body weight) and cyclosporine A $250 \mathrm{mg}$ daily (4.3 mg/kg body weight). Significant improvement was observed after 3 weeks of treatment. Complete remission of mucosal and skin lesions was achieved after a 6-month therapy.

According to available literature, a strong correlation between PSV and inflammatory bowel disease can be confirmed. Results of the retrospective analysis of all cases of PSV described in Mayo Clinic between 1995 and 2014 showed that PSV coexisted with inflammatory bowel disease (either Crohn's disease or UC) in all patients [5]. Moreover, PSV can be considered a marker of inflammatory bowel disease activity [6]. These findings are consistent with the case of our patient where mucosal lesions and UC exacerbation occurred concurrently. Pyostomatitis vegetans may affect patients at any age, but it is most commonly observed in ones between 20 and 59 years. Males show a considerable predominance over females with an incidence ratio of $2: 1-3: 1$, respectively $[7,8]$. Characteristic features of PSV clinical presentation include mucosal ulcerative lesions, pustules 

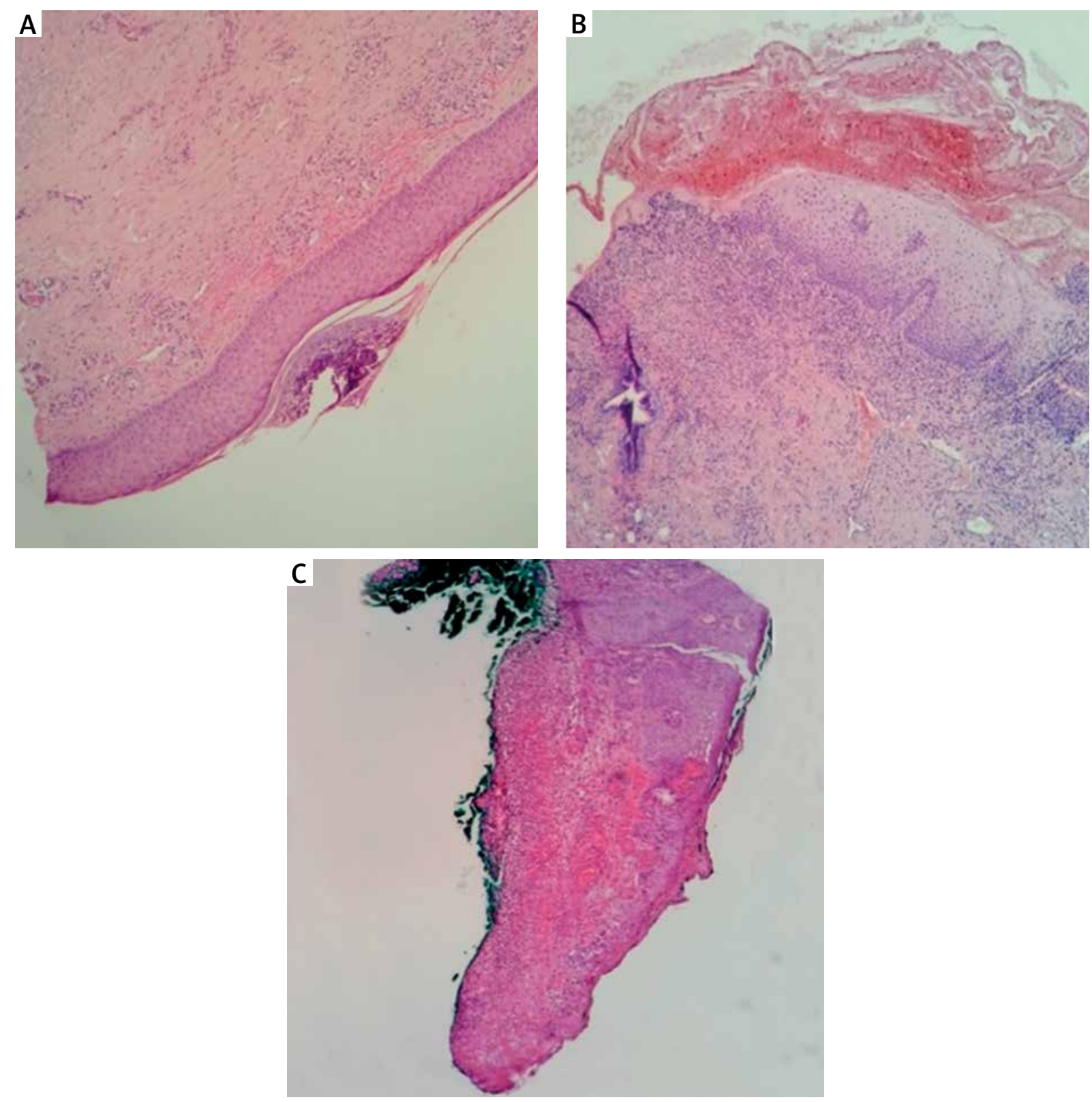

Figure 3. Histopathological features of initial phase mucosal lesions - mucosal pustules and erosions (A), advanced phase mucosal lesions - mucosal ulcerations (B), skin ulceration (C)

with mucosal erythema and oedema at the base. Major histopathological microscopic features of PSV comprise formation of intra-epithelial or sub-epithelial micro-abscesses as well as neutrophilic and eosinophilic infiltration. Additionally, hyperkeratosis and acanthosis can be found $[9,10]$. These abnormalities could be observed in our patient's case before the mucosal ulcerations appeared. Direct immunofluorescence for IgA, IgG and C3 deposits is negative in PSV, which allows to distinguish PSV from blistering autoimmune diseases [11]. Peripheral blood eosinophilia is a common but not mandatory abnormality described in PSV. In the aforementioned research by Clark et al., eosinophilia was present in 3 out of 7 patients with PSV [5], whereas other authors indicate that the rate of eosinophilia in the course of PSV can be much higher, even up to $90 \%$ [12]. There was no eosinophilia in the presented case.

Differential diagnosis of PSV should include blistering autoimmune diseases (pemphigus vulgaris, pemphigus paraneoplasticus, bullous pemphigoid, acquired epidermolysis bullosa), bullous drug eruptions, erythema multiforme, Stevens-Johnson syndrome, toxic epidermal 
Table 1. Differential diagnosis of pyostomatitis vegetans

\begin{tabular}{ll}
\hline Disease & Major typical features \\
\hline Blistering autoimmune diseases & Positive direct immunofluorescence test \pm indirect immunofluorescence test \\
\hline Chronic ulcerative stomatitis & Chronic condition, SES-ANA present in direct and/or indirect immunofluorescence test \\
\hline Behçet's disease & $\begin{array}{l}\text { International Study Group Criteria for Behçet's disease (recurrent oral ulcerations + 2 of the } \\
\text { following: recurrent genital ulcerations, eye lesions, skin lesions, positive pathergy reaction) }\end{array}$ \\
\hline Erosive lichen planus & $\begin{array}{l}\text { Histopathological features typical of lichen planus } \\
\text { Skin and nails lesions typical of lichen planus }\end{array}$ \\
\hline Systemic lupus erythematosus & $\begin{array}{l}\text { Systemic Lupus International Collaborating Clinics Classification Criteria for Systemic Lupus } \\
\text { Erythematosus [13] }\end{array}$ \\
\hline Herpetic stomatitis & Early age at onset, short course, spontaneous recovery, positive herpes testing \\
\hline Aphthae & Recurrent episodes, spontaneous recovery \\
\hline Systemic Vasculitis -Wegener's & $\begin{array}{l}\text { Typical histopathological presentation, organ disorders (especially lungs, kidneys), positive ANCA } \\
\text { granulomatosis }\end{array}$ \\
\hline Malignant neoplasms & Typical histopathological features, medical history and physical examination findings \\
\hline
\end{tabular}

necrolysis, chronic ulcerative stomatitis, Behçet's disease, erosive lichen planus, systemic lupus erythematosus, herpetic stomatitis, Wegener's granulomatosis and malignant neoplasms. Typical features of these diseases are presented in Table 1 [13].

In our patient's case, shortly after the excision of previously diagnosed melanoma, skin and mucosal lesions typical of PG appeared. Pyoderma gangrenosum most often coexists with PSV as its vegetating presentation (pyodermatitis vegetans). However, our patient presented with typical PG lesions. It remains unknown whether the procedure of melanoma excision or the melanoma itself should be considered as a triggering factor of PG appearance.

\section{Conflict of interest}

The authors declare no conflict of interest.

\section{References}

1. Burgdorf HC, Plewig G, Wolff HH, et al. Braun-Falco. Dermatologia. Wydawnictwo Czelej 2011.

2. Rudnicka L. Neutrophilic diseases and their overlap with autoinflammatory disease 24 $4^{\text {th }}$ EADV Congress 7 - 11 Oct 2015 Copenhagen, Denmark.

3. Romańska-Gocka K, Cieścińska C, Zegarska B, et al. Pyoderma gangrenosum with monoclonal IgA gammopathy and pulmonary tuberculosis. Illustrative case and review. Postep Dermatol Alergol 2015; 32: 137-41.

4. Nico MM, Hussein TP, Aoki V, et al. Pyostomatitis vegetans and its relation to inflammatory bowel disease, pyoderma gangrenosum, pyodermatitis vegetans, and pemphigus. J Oral Pathol Med 2012; 41: 584-8.

5. Clark LG, Tolkachjov SN, Bridges AG, et al. Pyostomatitis vegetans (PSV)-pyodermatitis vegetans (PDV): a clinicopathologic study of 7 cases at a tertiary referral center. J Am Acad Dermatol 2016; 75: 578-84.
6. Lankarani KB, Sivandzadeh GR, Hassanpour S. Oral manifestation in inflammatory bowel disease: a review. World J Gastroenterol 2013; 19: 8571-9.

7. Ruiz-Roca JA, Berini-Aytés L, Gay-Escoda C. Pyostomatitis vegetans. Report of two cases and review of the literature. Oral Surg Oral Med Oral Pathol Oral Radiol Endod 2005; 99 : 447-54.

8. Hegarty AM, Barrett AW, Scully C. Pyostomatitis vegetans. Clin Exp Dermatol 2004; 29: 1-7.

9. Femiano F, Lanza A, Buonaiuto C, et al. Pyostomatitis vegetans: a review of the literature. Med Oral Patol Oral Cir Bucal 2009; 14: 114-7.

10. Nico MM, Hussein TP, Aoki V, et al. Pyostomatitis vegetans and its relation to inflammatory bowel disease, pyoderma gangrenosum, pyodermatitis vegetans, and pemphigus. J Oral Pathol Med 2012; 41: 584-8.

11. Lourenço SV, Hussein TP, Bologna SB, et al. Oral manifestations of inflammatory bowel disease: a review based on the observation of six cases. J Eur Acad Dermatol Venereol 2010; 24: 204-7.

12. Matias Fde A, Rosa DJ, Carvalho MT, et al. Pyodermatitispyostomatitis vegetans: case report and review of medical literature. An Bras Dermatol 2011; 86: 137-40.

13. Petri M, Orbai AM, Alarcón GS, et al. Derivation and validation of the Systemic Lupus International Collaborating Clinics classification criteria for systemic lupus erythematosus. Arthritis Rheum 2012; 64: 2677-86. 